\title{
A Microwave Dielectric Biosensor Based on Suspended Distributed MEMS Transmission Lines
}

\author{
Lijie Li, Member, IEEE, and Deepak Uttamchandani, Senior Member, IEEE
}

\begin{abstract}
Design and characterization of a miniature microwave dielectric biosensor based on distributed microelectromechanical systems (MEMS) transmission lines (DMTL) is reported in this paper. The biosensor has been realized by bonding the DMTL device with an acrylic fluidic channel. In order to demonstrate the sensing mechanism, the sensor is used to detect the small variation of the concentration of aqueous glucose solutions by measuring the electromagnetic resonant frequency shift of the device. It is observed from the results that the second notch of the reflection coefficient $\left(S_{11}\right)$ varies from 7.66 to $7.93 \mathrm{GHz}$ and the third notch of the reflection coefficient varies from 15.81 to 15.24 $\mathbf{G H z}$ when the concentration of the glucose solution ranges from 0 to $347 \mathrm{mg} / \mathrm{ml}$, which indicates that higher order notches have higher sensitivities if looking at the absolute change in frequency.
\end{abstract}

Index Terms-Biosensor, distributed microelectromechanical systems (MEMS) transmission lines (DMTL), glucose.

\section{INTRODUCTION}

D IELECTRIC sensors play an important role in many industrial areas such as biological, agriculture, and food industries as this technology is noninvasive compared with optical and chemical sensing mechanisms. It usually detects changes of humidity, temperature, and concentration of aqueous solutions by measuring changes of impedance. Many radio frequency (RF) and microwave devices or circuits have been used for sensor application in the past [1]-[10]. The detection of the permittivity of aqueous solutions at $10 \mathrm{GHz}$ was achieved with a microwave resonator composed of sapphire cylinder and a quartz plate [1]. A microwave dielectric measurement kit composed of a coaxial reflectometric sensor terminated by a metallic cylindrical cell to contain the liquid has been developed to detect the complex permittivity of the liquids under extreme conditions [2]. Using periodic structures to enhance the sensitivity of microwave planar sensors is an approach that has been theoretically proposed previously with an electromagnetic band gap (EBG) structure [3], where the basic principle is to reduce the wave group velocity to induce greater interaction between the sensor and the material under test (MUT). A microwave

Manuscript received June 11, 2009; revised July 13, 2009; accepted July 13, 2009. Current version published October 23, 2009. This work was supported in part by the Scottish Funding Council under Glasgow Research Partnership in Engineering Scheme, and Royal Society Research Grant RG080484. The associate editor coordinating the review of this paper and approving it for publication was Dr. Andreas Hierlemann.

The authors are with the Centre for Microsystems and Photonics, Department of Electronic and Electrical Engineering, University of Strathclyde, Glasgow, G1 1XW, U.K. (e-mail: lijie.li@ieee.org).

Color versions of one or more of the figures in this paper are available online at http://ieeexplore.ieee.org.

Digital Object Identifier 10.1109/JSEN.2009.2031388 resonator based on a coplanar waveguide (CPW)-to-slotline resonator ring has been reported to form a humidity sensor [4]. The concept of using CPW lines as dielectric sensors has been presented in reference [5]. A microwave biosensor for detecting the concentration of aqueous glucose solutions has been developed based on a cylindrical air gap coupled to a microstrip line at resonant frequency of $1.68 \mathrm{GHz}$ [6]. A gas sensor has been reported based on a micromachined membrane supported CPW structure filled with a mixture of carbon nanotube [7]. Based on CPW structures, a Goubau transmission line for biosensing has been reported in [8]. Microwave CPW structures have also been used as biosensors in the frequency range between $40 \mathrm{~Hz}$ to $26.5 \mathrm{GHz}$ in [9]. A particle sensing and cell counting system has been developed based on micromachined CPW structures in [10]. A comprehensive review on the microwave dielectric measurements on polar liquids has been reported previously [11].

Distributed microwave transmission lines are basically microwave transmission lines periodically loaded with active or passive electronic components, such as transistors or capacitors, are typical slow wave structures. They are usually used in the electronic applications such as amplifiers, oscillators, mixers, multipliers and phase shifting circuits. Distributed transmission lines incorporating MEMS have been realized in the past. Barker and Rebeiz [12] demonstrated a distributed MEMS transmission line (DMTL) phase shifter using MEMS capacitive switches. After their work, much effort has been directed on design, modification, and fabrication of DMTLs [13]-[21] for communication applications. Since the distributed transmission lines are classical slow-wave multiple resonant structures, which induces higher interaction between sensors and MUT, they are suitable to construct very sensitive biosensors. Previously a MEMS DMTL biosensor fabricated using flip-chip technology based on a ceramic substrate has been demonstrated [22]. In this paper, a biosensor incorporating a microchannel fabricated with acrylic glass and a monolithic microfabricated DMTL device is reported.

As shown schematically in Fig. 1, the biosensor consists of two structures, the bottom structure is a distributed monolithic MEMS transmission line fabricated on a high resistivity silicon substrate. The top structure is a microchannel structure fabricated on acrylic material. The two structures are joined together with strain gauge cement. The sensing mechanism is based on the electromagnetic interaction between a biosample inside the channel and the microwave signal propagating through the DMTL device. Different concentrations of aqueous glucose solutions were used to characterize the biosensor, and the results are reported in this paper. In the experiment, the liquid is pumped into the channel with a standard $5 \mathrm{ml}$ syringe. In 

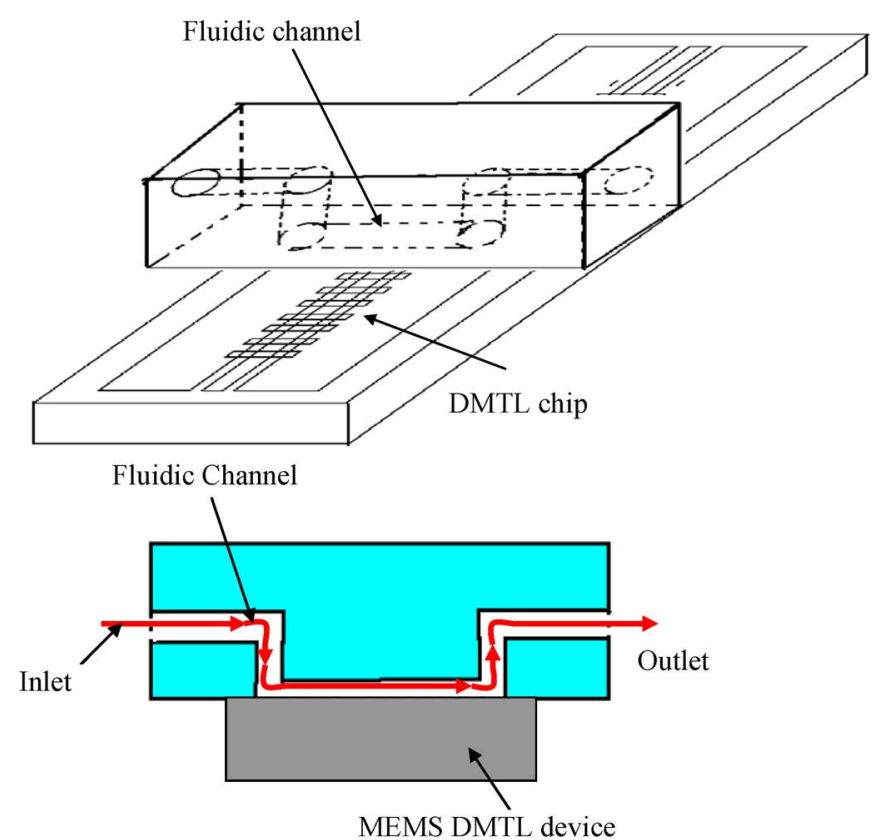

Fig. 1. Schematic of the DMTL biochip. Top figure is the 3D view and bottom is the cross-section view.

the next section, the design and fabrication of the biosensor is described in detail, after which the low-frequency to microwave-frequency measurements and analysis of the DMTL sensor with fluidic samples are presented.

\section{Design AND FABRICATION OF DMTL BIOSENSOR}

\section{A. Design and Modeling}

The DMTL device has been realised by a commercial MEMS foundry process-MetalMUMPs. The process uses high resistivity silicon as the substrate in order to reduce electromagnetic loss through the substrate. For the coplanar waveguide, the signal and ground lines of the coplanar waveguide (CPW) are constructed using a layer of nickel with thickness of $20 \mu \mathrm{m}$. The waveguide is suspended above the silicon substrate with a $25 \mu \mathrm{m}$ air gap. The $0.7 \mu \mathrm{m}$ polysilicon capacitor bridge is situated under the nickel waveguide with a $1.1 \mu \mathrm{m}$ air gap. The width of the signal line and the gap between signal line and ground plane is designed to be 40 and $43 \mu \mathrm{m}$ respectively. As the CPW is on a double-layer dielectric substrate [Fig. 2(a)], the characteristic impedance and the effective relative dielectric constant $\varepsilon_{\text {eff }}$ are calculated according the formulas below [23]

$$
\begin{aligned}
Z_{0}= & \frac{30 \pi}{\sqrt{\varepsilon_{\text {eff }}}} \frac{K\left(k_{0}^{\prime}\right)}{K\left(k_{0}\right)} \\
\varepsilon_{\text {eff }}= & 1+\frac{\left(\varepsilon_{1}-1\right)}{2} \frac{K\left(k_{1}\right)}{K\left(k_{1}^{\prime}\right)} \frac{K\left(k_{0}^{\prime}\right)}{K\left(k_{0}\right)} \\
& +\frac{\left(\varepsilon_{a i r}-\varepsilon_{1}\right)}{2} \frac{K\left(k_{2}\right)}{K\left(k_{2}^{\prime}\right)} \frac{K\left(k_{0}^{\prime}\right)}{K\left(k_{0}\right)} \\
k_{0}= & \frac{W}{L}, \quad k_{0}^{\prime}=\sqrt{1-k_{0}^{2}}
\end{aligned}
$$

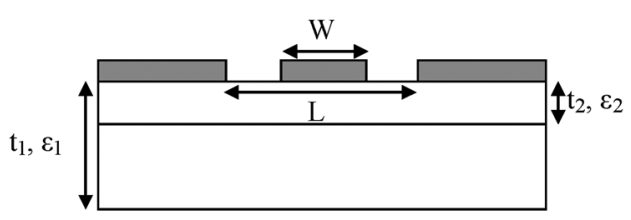

(a)

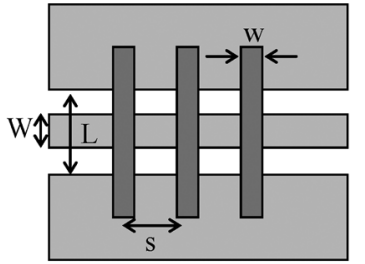

(b)

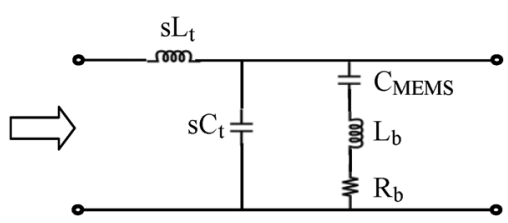

(c)
Fig. 2. Schematic diagram of the DMTL and its equivalent circuit.

$$
\begin{array}{ll}
k_{1}=\frac{\sinh \left(\frac{\pi W}{4 t_{1}}\right)}{\sinh \left(\frac{\pi L}{4 t_{1}}\right)}, & k_{1}^{\prime}=\sqrt{1-k_{1}^{2}} \\
k_{2}=\frac{\sinh \left(\frac{\pi W}{4 t_{2}}\right)}{\sinh \left(\frac{\pi L}{4 t_{2}}\right)}, & k_{2}^{\prime}=\sqrt{1-k_{2}^{2}}
\end{array}
$$

where $Z_{0}$ is the characteristic impedance of the $\mathrm{CPW}, \varepsilon_{e f f}$ is the effective dielectric constant of the CPW, $K(k)$ is the complete elliptic integral of the first kind, $t_{1}$ and $t_{2}$ are the thicknesses of the silicon substrate and air gap, $W$ and $L$ are the centre conductor width and total width of the CPW line, respectively, as shown in Fig. 2. The relative dielectric constant of the silicon layer $\varepsilon_{1}$ is 11.9 , and the relative dielectric constant of the air $\varepsilon_{\text {air }}$ is 1 . The width of the central conductor $(W)$ is designed as $40 \mu \mathrm{m}$, and the gap between central conductor and ground plane is designed as $43 \mu \mathrm{m}$. The $\varepsilon_{\text {eff }}$ and $Z_{0}$ are calculated to be around 3.2 and $88.4 \Omega$ using (1)-(5). The per unit-length (the unit is meter) capacitance $C_{t}$ and inductance $L_{t}$ of the unloaded CPW transmission line are given by [12]

$$
C_{t}=\frac{\sqrt{\varepsilon_{\text {eff }}}}{c Z_{0}}, \quad L_{t}=C_{t} Z_{0}^{2}
$$

where $c$ is the free space electromagnetic wave velocity. From (6), $C_{t}$ and $L_{t}$ are calculated to be $67.5 \mathrm{pF}$ and $526.9 \mathrm{nH}$, respectively.

The CPW line periodically loaded with capacitors can be modeled as a lumped inductance $\left(L_{t}\right)$ and a lumped capacitance $\left(C_{t}\right)$ with a parallel capacitor to ground due to the polysilicon bridges $\left(C_{\text {MEMS }}\right)$, as schematically shown in Fig. 2(c). Ignoring bridge inductance, $L_{b}$ and the bridge resistance $R_{b}$, the characteristic impedance $Z_{l}$, and the Bragg frequency $f_{B r a g g}$ are given by [12]

$$
\begin{aligned}
Z_{l} & =\sqrt{\frac{L_{t}}{C_{t}+C_{\mathrm{MEMS}} / s}} \\
f_{\mathrm{Bragg}} & =\frac{1}{\pi \sqrt{s L_{t}\left(s C_{t}+C_{\mathrm{MEMS}}\right)}}
\end{aligned}
$$




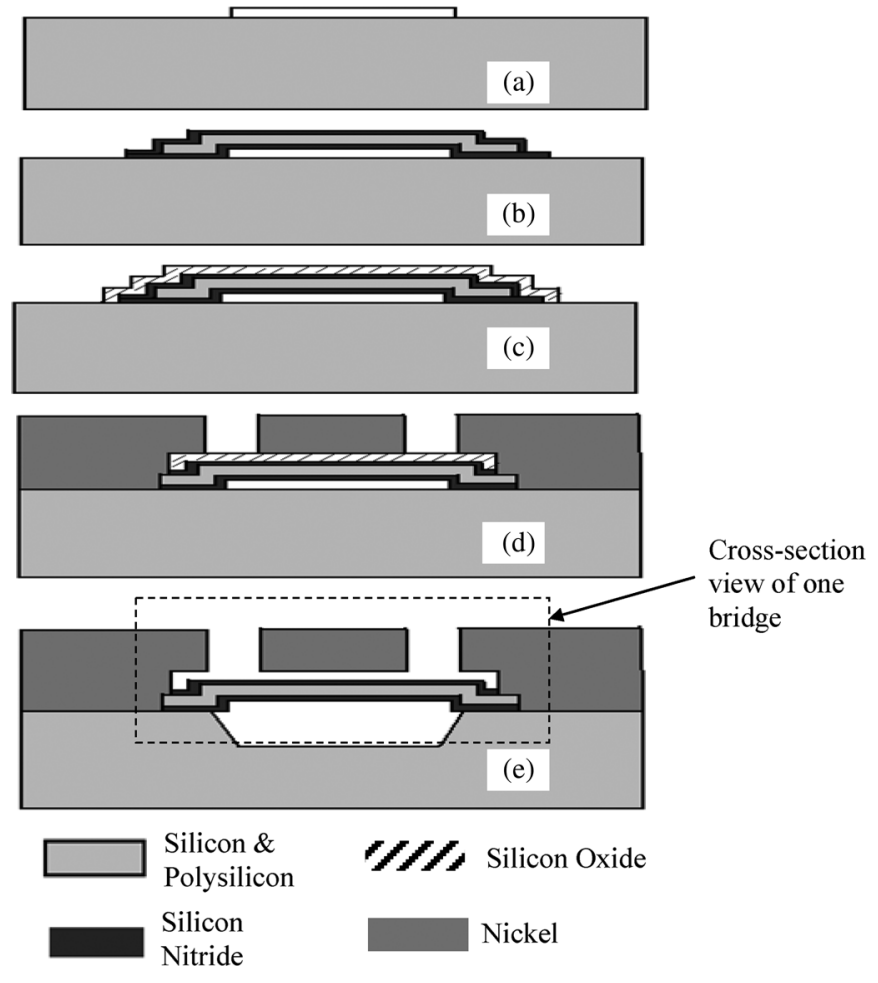

Fig. 3. Process flow.

where $s$ is the periodic spacing between the suspended bridges of the DMTL. The Bragg frequency is the frequency at which the characteristic impedance of the DMTL goes to zero, indicating no power transfer. The $Z_{l}$ and $f_{\text {Bragg }}$ of this design are calculated to be $77.5 \Omega$ and $156 \mathrm{GHz}$. The equivalent model, i.e., Fig. 2(c) based on extracted/calculated electronic components for analysing DMTL devices is generally acceptable and has been used in most previous publications [13]-[21]. The equivalent model of the unloaded DMTL has been built and the simulated S-parameters will be shown in the Section III.

\section{B. Fabrication}

The monolithic DMTL device was fabricated with multilayer MEMS foundry process-MetalMUMPs. The fabrication process of the DMTL device is summarized as follows (shown in Fig. 3): a high resistivity $(\sim 5000 \mathrm{O}-\mathrm{cm})$ silicon wafer is used as the starting substrate; a layer of silicon oxide is then deposited and patterned. This oxide layer outlines the area that will be used to etch a trench in the silicon substrate. A layer of silicon nitride is deposited, followed by the deposition of a layer of polysilicon, which will be used to define membrane capacitor. A second layer of silicon nitride is then deposited to protect the polysilicon film from being etched in the trench formation process. A second silicon oxide layer is then deposited and this will be sacrificially removed to leave an air gap between membrane capacitor and CPW waveguide. A metal layer consisting of $20 \mu \mathrm{m}$ of electroplated nickel with $0.5 \mu \mathrm{m}$ of gold deposited on top of the nickel layer is used for forming the $\mathrm{CPW}$. The last step in the process is to etch out the sacrificial
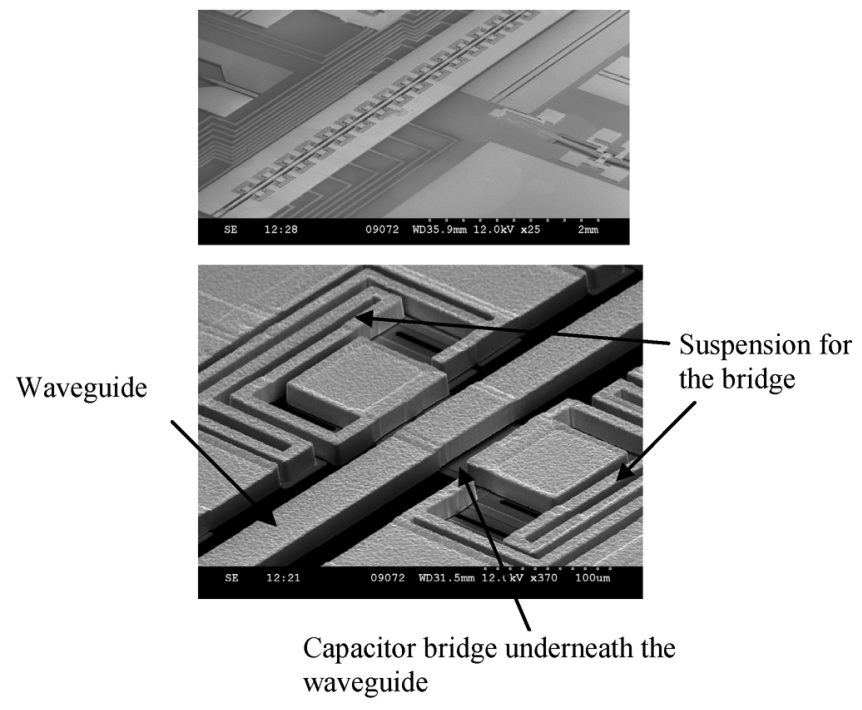

Fig. 4. SEM of the fabricated device.

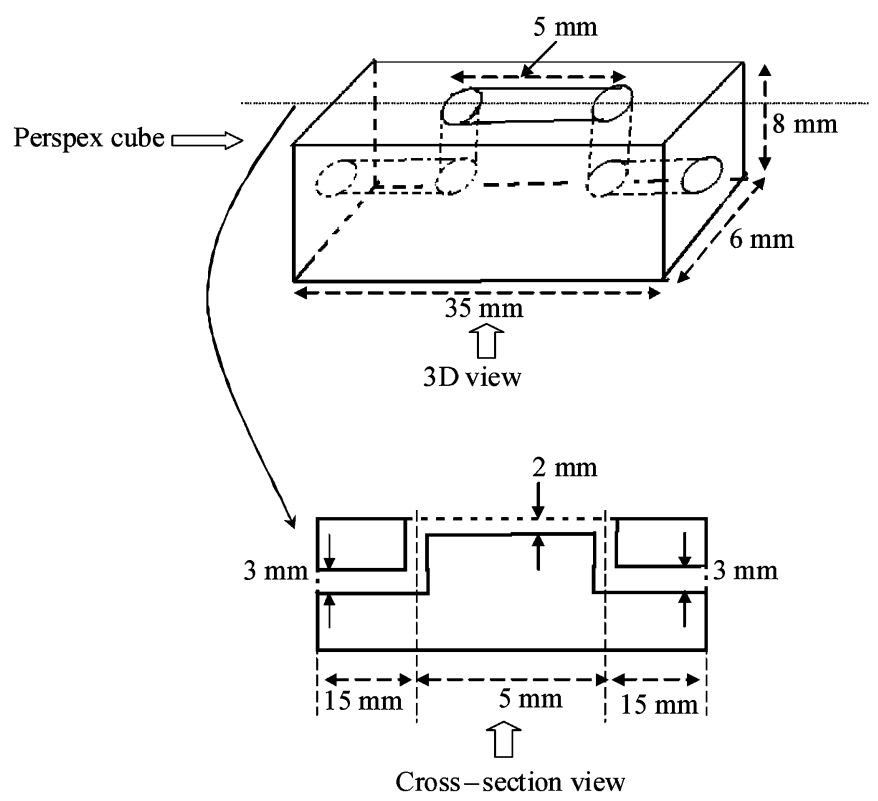

Fig. 5. Schematic and dimensions of the microchannel.

layers as well as to etch a $25 \mu \mathrm{m}$ trench in the silicon substrate. The trench etch of the substrate is determined by the first oxide layer. Full details of the MetalMUMPs process, together with Design Rules can be obtained from [9]. The scanning electron micrograph of the fabricated DMTL device is shown in Fig. 4.

After fabricating the DMTL device, a microchannel was machined on an acrylic substrate, and the dimensions of the microchannel device are shown in Fig. 5. Finally, the DMTL device and microchannel are bonded using KYOWA CC-33A strain gage cement. The photograph of the completed biosensor is shown in Fig. 6. Short plastic pipes (3 $\mathrm{mm}$ in diameter) are fixed onto the inlet and outlet of the microchannel to allow the biosample to be fed in. The fluidic sample was pumped in using a standard syringe. 

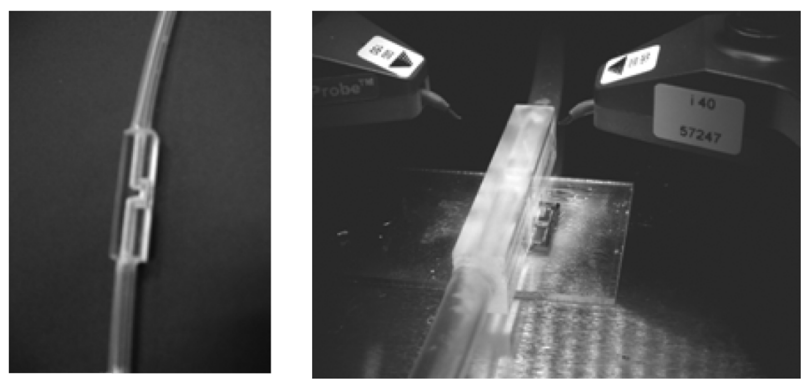

Fig. 6. Left photograph shows a microchannel device with plastic tube; right photograph shows the completed biosensor device under a GSG probe station.

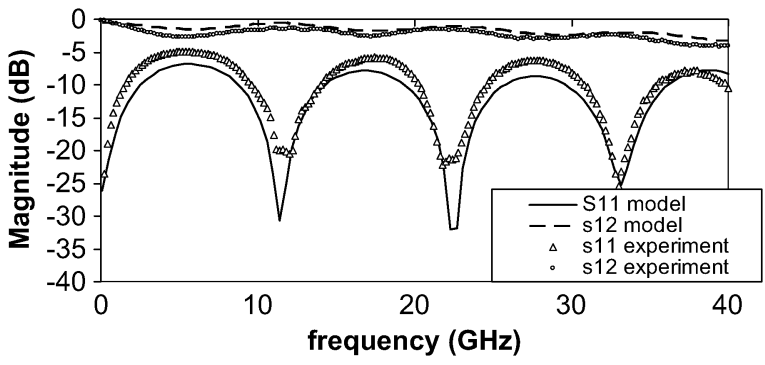

Fig. 7. Microwave characteristics of the DMTL device.

\section{MEASUREMENT}

\section{A. Microwave Measurement of the DMTL Chip}

The microwave characteristics of the DMTL device has been measured before assembling with the microchannel using a Cascade Microtech 9000 GSG probe station and an Agilent N5230A vector network analyzer. Before microwave measurement, Line-Reflect-Reflect-Match (LRRM) calibration technique has been applied using standard calibration substrate that came with the probe station. The technique is straight forward by following the automatic instruction from the software. GSG probe was sequentially landed on a standard short, a standard open, and a standard $50 \mathrm{Ohm}$ line to complete the calibration procedure. The two-port calibrated measurement of the DMTL device has been obtained, and the reflection coefficient S11 and transmission coefficient S12 are shown in Fig. 7. It is seen from S11 that there are four notches in the frequency range of $0-40 \mathrm{GHz}$ including the one in low-frequency. The circuit model was built based on the calculated lumped parameters of the transmission line described in Section II. EM3DS circuit solver v1.0 has been used to construct the model. The model basically contains 19 section connected in series, each of which has been modeled according Fig. 2(c). The capacitance of the MEMS bridge structure has been experimentally extracted to be around $6 \mathrm{fF}$. Series resistance of the polysilicon bridge was taken into account with estimated value of $1 \mathrm{Ohm}$. The modeled results are also shown in the graph for comparison.

\section{B. Microwave Measurement of the Biological Sample}

After the assembling of the biosensor was completed, DI water and aqueous glucose solutions of different concentrations were injected into the channel. Reflection coefficient S11 was measured in various conditions. What is known to us that

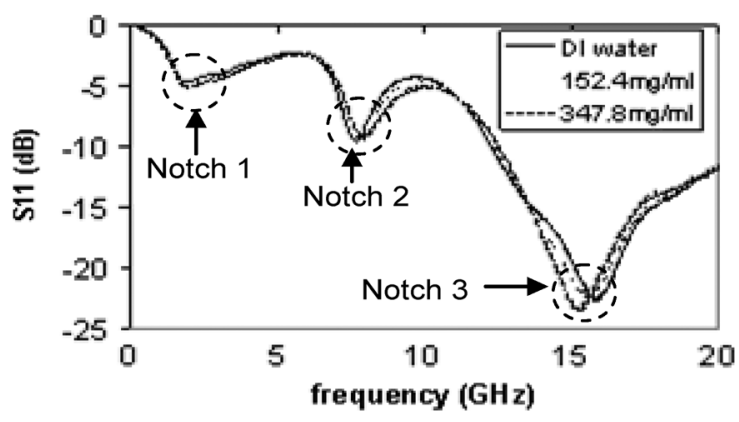

Fig. 8. Microwave measurement of the glucose solution with different concentrations.

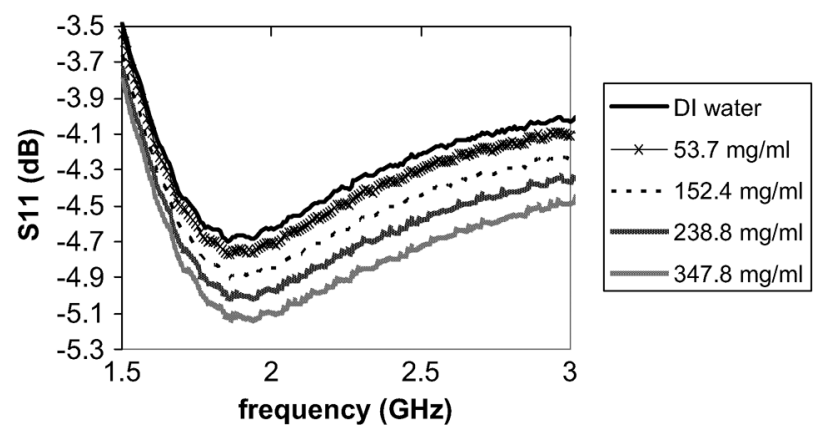

Fig. 9. Frequency changing at the first notch

the conducting aqueous solutions (there are free ions in the solution) can be precisely detected at the low-frequency range of the DMTL biosensors [22]. In principle, the nonconducting solutions (such as glucose solutions) can be detected at higher frequencies. The advantage of using periodic structure is that there are many notches in the wide frequency band, and very high resolutions can be achieved at higher frequency notches. Reflection coefficient S11 of the biosensors loaded with aqueous glucose solutions of various concentrations ranging from 0 to $350 \mathrm{mg} / \mathrm{ml}$ were measured, and the results are shown in Fig. 8. The aqueous glucose solution was prepared using deionized water with the glucose powder. It is shown that there are three notches in $0-20 \mathrm{GHz}$ frequency band. Very minor differences can be seen in the first notch $(1.8 \mathrm{GHz})$, and that is getting obvious at second notch $(7.7 \mathrm{GHz})$. At the third notch (around $16 \mathrm{GHz}$ ), it is clearly seen that as the concentration gets higher, the resonant peak moves from high to low values. In order to investigate this in detail, the 1-3 notches are picked up individually and shown in Figs. 9-11.

It is seen from Fig. 9 that as the concentration of the glucose solution changes, the resonant frequency changes slightly from lower to higher values. Since the $\mathrm{Q}$ factor at the first notch is very low, it is very hard to obtain any reliable readings. The changes of resonant frequency upon different concentrations of the solution are getting obvious at the second notch (resonant frequency changes from 7.66 to $7.93 \mathrm{GHz}$ as the concentration of the solution varies from 0 to $347.8 \mathrm{mg} / \mathrm{ml}$ ). The resonant frequency at third notch becomes more sensitive to the concentration of the glucose solution (resonant frequency changes from 15.81 to $15.24 \mathrm{GHz}$ as the concentration of the solution varies from 0 to $347.8 \mathrm{mg} / \mathrm{ml})$. $\Delta \mathrm{f}$ at second and third notches versus concentration of the glucose solution is shown in Fig. 12. It is 


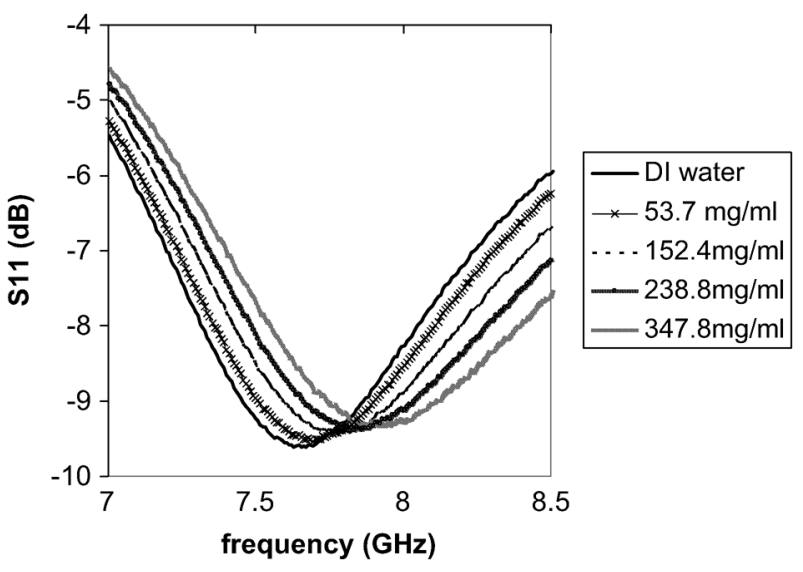

Fig. 10. Frequency changing at the second notch.

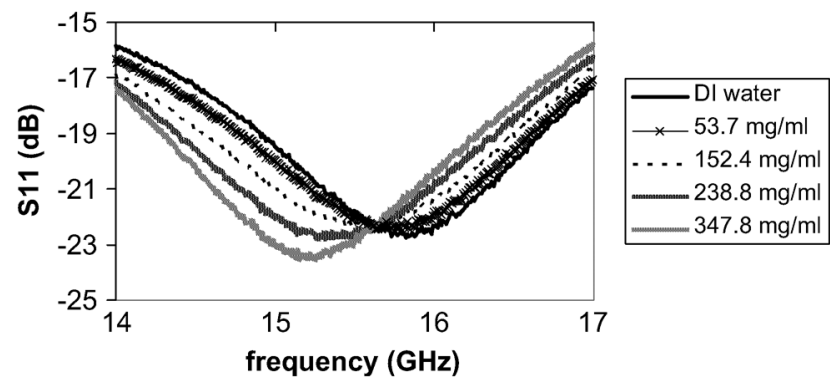

Fig. 11. Frequency changing at the third notch.

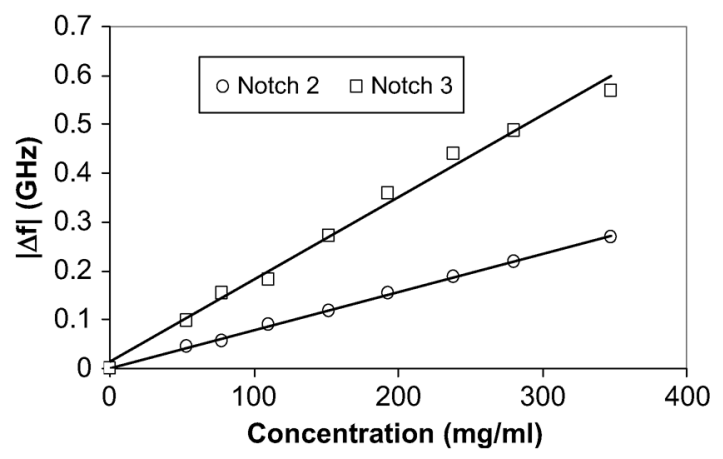

Fig. 12. $|\Delta t|$ versus concentration of the glucose solution.

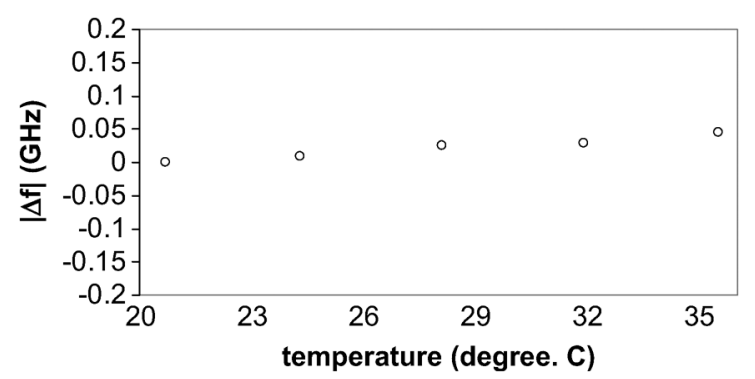

Fig. 13. $|\Delta t|$ versus temperature, the concentration of the glucose solution is $142.8 \mathrm{mg} / \mathrm{ml}$.

shown that: (a) the frequency varies linearly with the concentration and (b) absolute changes in frequency at notch 3 are more than double of the resonant changes at notch 2 .

The effect on the biosensor due to different temperatures has been studied using preheated glucose solutions. The con-

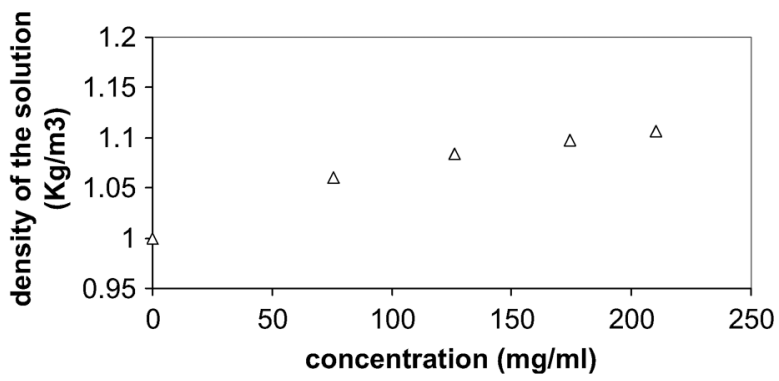

Fig. 14. Concentration versus density for glucose solution.

centration of the glucose solution was chosen randomly at $142.8 \mathrm{mg} / \mathrm{ml}$. It is seen from the result shown in Fig. 13, the $\Delta f$ increases slightly as the temperature of the solution rises from 20 to 35 Celsius. Since there is no in situ temperature sensor integrated with the biosensor, the precise temperature measurement cannot be obtained. However, the result does tell the trend of the temperature dependent performance of the sensor. In the future design, an in situ polysilicon temperature sensor will be designed with the biosensor for precisely extracting the temperature dependent performance of the device.

As it is known previously, the density of the solution increases when the concentration of the glucose solution increases. A simple experiment has been performed to measure the density of the different concentrations. The result is shown in Fig. 14. It is shown that the density varies from 1 to $1.1 \mathrm{~kg} / \mathrm{m}^{3}$ as the concentration of the glucose solution changes from 0 to $210 \mathrm{mg} / \mathrm{ml}$.

\section{CONCLUSION}

In this paper, a biosensor that is based on a distributed MEMS transmission line has been presented. The biosensor was assembled with a DMTL chip and a microchannel device that allows biosamples to interact with the electromagnetic field through the DMTL. The MEMS chip was designed and fabricated using a commercial foundry process. Both the DMTL device and assembled biosensor have been measured in a wide frequency range $(0-40 \mathrm{GHz})$. The measurement of the DMTL device shows a good agreement with the lumped element modeling. The RF measurement of the biosensor using aqueous glucose solutions shows that frequency changes are greater in the higher order notches.

\section{REFERENCES}

[1] E. N. Shaforost, N. Klein, S. A. Vitusevich, A. A. Barannik, and N. T. Cherpak, "High sensitivity microwave characterization of organic molecule solutions of nanoliter volume," Appl. Phys. Lett., vol. 94, p. $112901,2009$.

[2] G. A. Dimitrakis, M. George, M. Poliakoff, I. Harrison, J. Robinson, S Kingman, E. Lester, A. P. Gregory, and K. Lees, "A system for traceable measurement of the microwave complex permittivity of liquids at high pressures and temperatures," Measurement Sci. Technol., vol. 20, p. $045901,2009$.

[3] B. Garcia-Banos, F. Cuesta-Soto, A. Griol, J. M. Catala-Civera, and J. Pitarch, "Enhancement of sensitivity of microwave planar sensors with EBG structures," IEEE Sensors J., vol. 6, no. 6, pp. 1518-1522, Jun. 2006.

[4] Y. H. Kim, K. Jang, Y. J. Yoon, and Y. J. Kim, "A novel relative humidity sensor based on microwave resonators and a customized polymeric film,” Sens. Actuators B, vol. 117, pp. 315-322, 2006. 
[5] S. S. Stuchly and C. E. Bassey, "Microwave coplanar sensors for dielectric measurements," Measurement Sci. Technol., vol. 9, pp. 1324-1329, 1998.

[6] J. Kim, A. Babajanyan, A. Hovsepyan, K. Lee, and B. Friedman, "Microwave dielectric resonator biosensor for aqueous glucose solution," Rev. Sci. Instrum., vol. 79, p. 086107, 2008.

[7] M. Dragoman, K. Grenier, D. Dubuc, L. Bary, and R. Plana, "Millimeter wave carbon nanotube gas sensor," J. Appl. Phys., vol. 101, p. $106103,2007$.

[8] A. Treizebre, T. Akalin, and B. Bocquet, "Planar excitation of Goubau transmission lines for THz bioMEMS," IEEE Microw. Wireless Components Lett., vol. 15, no. 12, pp. 886-888, 2005.

[9] G. R. Facer, D. A. Notterman, and L. L. Sohn, "Dielectric spectroscopy for bioanalysis: From $40 \mathrm{~Hz}$ to $26.5 \mathrm{GHz}$ in a microfabricated wave guide," Appl. Phys. Lett., vol. 78, no. 7, pp. 996-998, 2001.

[10] D. K. Wood, S. H. Oh, S. H. Lee, H. T. Soh, and A. N. Cleland, "Highbandwidth radio frequency coulter counter," Appl. Phys. Lett., vol. 87, p. $184106,2005$.

[11] A. P. Gregory and R. N. Clark, "A review of RF and microwave techniques for dielectric measurements on polar liquids," IEEE Trans. Dielectr. Electr. Insul., vol. 13, no. 4, pp. 727-743, 2006.

[12] N. S. Barker and G. M. Rebeiz, "Distributed MEMS true-time delay phase shifters and wide-band switches," IEEE Trans. Microw. Theory Tech., vol. 46, no. 11, pp. 1881-1890, Nov. 1998.

[13] N. S. Barker and G. M. Rebeiz, "Distributed MEMS transmission line BPSK modulator," IEEE Microw. Guided Wave Lett., vol. 10, no. 5, pp. 198-200, May 2000.

[14] Y. Liu, A. Borgioli, A. S. Nagra, and R. A. York, "Distributed MEMS transmission lines for tunable filter applications," Int. J. RF and Microw. Comput.-Aided Eng., vol. 11, no. 5, pp. 254-260, Aug. 2001.

[15] Q. Shen and N. S. Barker, "Distributed MEMS tunable matching network using minimal-contact RF-MEMS varactors," IEEE Trans. Microw. Theory Tech., vol. 54, no. 6, pp. 2646-2658, Jun. 2006.

[16] J. S. Hayden and G. M. Rebeiz, "Very low-loss distributed X-band and Ka-band MEMS phase shifters using metal-air-metal capacitors," IEEE Trans. Microw. Theory Tech., vol. 51, pp. 309-314, Jan. 2003.

[17] J. J. Hung, L. Dussopt, and G. M. Rebeiz, "Distributed 2- and 3-bit W-band MEMS phase shifters on glass substrates," IEEE Trans. Microw. Theory Tech., vol. 52, pp. 600-606, Feb. 2004.

[18] S. Afrang and B. Y. Majlis, "Distributed transmission line phase shifter using MEMS switches and inductors," Microsystem Technologies-Micro-and Nanosystems-Information Storage and Process. Syst., vol. 14, pp. 1173-1183, 2008.

[19] K. Topalli, M. Unlu, and S. Demir, "New approach for modelling distributed MEMS transmission lines," IEE Proc. Microw. Antennas Propagat., vol. 153, pp. 152-162, 2006.
[20] J. Perruisseau-Carrier, R. Fritschi, P. Crespo-Valero, and A. K. Skrivervik, "Modeling of periodic distributed MEMS-Application to the design of variable true-time delay lines," IEEE Trans. Microw. Theory Tech., vol. 54, pp. 383-392, 2006.

[21] W. Palei, A. Q. Liu, A. B. Yu, A. Alphones, and Y. H. Lee, "Optimization of design and fabrication for micromachined true time delay (TTD) phase shifters," Sens. Actuators A, vol. 119, pp. 446-454, 2005.

[22] L. Li and D. Uttamchandani, "Flip-chip distributed MEMS transmission lines for biosensing applications," IEEE Trans. Ind. Electron., vol. 56, no. 4, pp. 986-990, 2009.

[23] R. N. Simons, Coplanar Waveguide Circuits, Components, and Systems. New York: Wiley, 2001.

Lijie Li (M'07) received the Ph.D. degree in design, modeling, and characterization of microactuators and optical MEMS devices from the Centre for Microsystems and Photonics, Department of Electronic and Electrical Engineering, University of Strathclyde, Glasgow, U.K., in 2004.

From 2006 to August 2007, he was a Senior MEMS Design Engineer at the Institute of System Level Integration, U.K. He is now a Lecturer at the Centre for Microsystems and Photonics, Department of Electronic and Electrical Engineering, University of Strathclyde. He is author and coauthor of over 60 scientific papers and one U.K. patent in the field of MEMS. His current research interests are in microsensors and actuators, optical microsystems, radio frequency microsystems, BioMEMS, high-power MEMS, CMOS IC design and characterization, and integrated microsystems.

$\mathrm{Dr}$. $\mathrm{Li}$ is a member of the Technical Committee in MEMS in the IEEE Industrial Electronics Society.

Deepak Uttamchandani (M'83-SM'03) received the Ph.D. degree from the University College London, London, U.K., in 1985.

$\mathrm{He}$ is a Professor of Microsystems Engineering, the Director of the Centre for Microsystems and Photonics, Department of Electronic and Electrical Engineering (EEE), the Vice Dean of the Engineering Faculty, Strathclyde University, Glasgow, Scotland, U.K. He is the Founder and the Editor-in-Chief of Institution of the Engineering and Technology (IET) Micro and Nano Letters. His current research interests include application of photonic, microsystems, and microelectromechanical systems (MEMS) technologies to optoelectronic sensors and systems such as optically excited micromechanical resonator sensors, mechanical characterization of MEMS materials, and photonic and RF applications of MEMS technology. 